\title{
A New Test Sequence Generation Method for Interoperability Testing
}

\author{
Naonobu OKAZAKI $\dagger 1$, Mi Rang PARK $\dagger \dagger 2$, \\ Kaoru TAKAHASHI ${ }^{\dagger}$ and Norio SHIRATORI ${ }^{\dagger} \dagger$
}

†Advanced Intelligent Communication System Laboratories, Ltd.

6-6-3, Minami-yoshinari, Aoba-ku, Sendai, 989-32 JAPAN

Phone : +81-22-279-3310 Fax : +81-22-279-3640 E-mail : oka@aic.co.jp

$\dagger+$ Research Institute of Electrical Communication, TOHOKU UNIVERSITY Aoba-ku, Sendai, 980 JAPAN

Phone : +81-22-227-6200 Ex.3412 Fax : +81-22-212-1439

\begin{abstract}
A method for generating test sequences for interoperability testing of communication systems is proposed. In the proposed method, the system state graph, which shows behaviours of the whole system, is generated from protocol specifications, and test sequences that confirm each transition and the state based on this graph are generated. The method is characterized in that the states of individual processes are confirmed, considering independency of the two processes, which is a characteristic of the interoperability testing. An application for a simple protocol with a retransmission mechanism shows that we can improve testing reliability in interoperability testing. Using this method is expected to decrease cost for generating test sequences.
\end{abstract}

\section{Introduction}

Testing is one of the major problem on developing communication software, and test sequence generation is a major part of it. Currently, as testings for communications systems, combinations of "conformance testing" and "interoperability testing" are being performed. About conformance testing[1], there are some studies on FSM(Finite State Machine) based test sequence generation[2]-[5]. Regarding these methods, Reference [2] called trace (based) method is based on tracing the FSM and References [3]-[5] called state oriented methods are based on identifying states in the FSM. It is shown in Reference [6] that a part of errors of the transition function that can be detected by test sequences generated by state oriented methods cannot be detected by the test sequences generated by trace method.

As a study on generation of test sequences for interoperability testing, Reference [10] shows a simple example of the method of generating test sequences using the reachability graph which shows the behaviours of the whole system. This method is

\footnotetext{
1 The auther is now with the Communication Processing Dept., Communication Systems Laboratory, MITSUBISHI Electric Corporation 5-1-1 Ofuna, Kamakura, 247 JAPAN

Phone : +81-467-41-2434 Fax : +81-467-41-2419 E-mail : oka@csl.melco.co.jp

2 The auther is now with the Advanced Switching Research Dept., Communication Systems Laboratory, MITSUBISHI Electric Corporation 5-1-1 Ofuna, Kamakura, 247 JAPAN

Phone : +81-467-41-2471 Fax : +81-467-41-2486 E-mail : mirang@csl.melco.co.jp
} 
based on tracing the reachability graph. There is a problem, however, in that as in the case of the above existing conformance test sequence generation method based on tracing, a part of errors of the transition function cannot be detected by test sequences generated by this method.

In this paper, a new method for generating interoperability test sequences having a high error-detecting capability by resolving the above problem of Reference [10] is proposed. In this method, the graph showing the behaviours of the whole system called the system state graph (SSG) is introduced and test sequences are generated on the bases of identification of the states on the SSG. At first, a method which applies the existing state oriented methods[3]-[5] for conformance testing to the SSG is presented, and its efficiency and limitation of this method are discussed. Next, in order to resolve the limitation of this method, a method for generating test sequences which identifies the states of individual processes, considering the independency of each process in interoperability testing, is given. To evaluate the error detection coverage of this method, a simple protocol with a retransmission mechanism is applied. It will show that we can improve testing reliability in interoperability testing.

The paper is organized as follows : Section 2 shows models of the tested communication systems and a testing environment. Section 3 contains a brief review of the concept of state identification of FSM and the existing conformance test sequence generation methods. The interoperability test sequence generation method proposed by this study and its application are presented in Section 4 . Conclusions are given in Section 5 .

\section{Modeling of Test Systems}

In this paper, the environment of the tested communication system under test is modeled as shown in Figure 1. In this model, a couple of protocol entities communicating with each other in the Nth layer, (N)-Entity 1 and (N)-Entity 2 are considered. The upper layer entities connected with (N)-Entity 1 and (N)-Entity 2 via (N)SAPs(Service Access Points) are called User 1 and User 2 respectively. (N)Entity 1 and (N)-Entity 2 are connected with two-directional FIFO(First In First Out) channels via (N-1)SAPs. Here, the protocol composed of the processes modeled by two communicating FSMs is defined as follows :

[Definition 1] Protocol $P=\left(\mathrm{P}_{1}, \mathrm{P}_{2}\right)$

Where,

$\mathrm{P}_{\mathrm{k}}: k$-th process $(k=1,2)$

$\mathrm{P}_{\mathrm{k}}=\left\langle\mathrm{Q}_{\mathrm{k}}, \mathrm{I}_{\mathrm{k}}, \mathrm{O}_{\mathrm{k}}, \omega_{\mathrm{k}}, \delta_{\mathrm{k}}, \mathrm{q}_{\mathrm{k} 0}\right\rangle$

$\mathrm{Q}_{\mathrm{k}}:$ states of $k$-th process

$\mathrm{I}_{\mathrm{k}}$ : input actions of $k$-th process

$\mathrm{I}_{\mathrm{k}}=\left\{p ? m_{\mathrm{i}} \mid p \in \mathrm{SAP}_{\mathrm{k}}, m_{\mathrm{i}} \in \mathrm{M}_{\mathrm{jk}} \cup \mathrm{M}_{\mathrm{k}_{\mathrm{i}}}\right\}$

$\mathrm{SAP}_{\mathrm{k}}: \mathrm{SAPs}$ of $k$-th process

$\mathrm{M}_{\mathrm{jk}}$ : messages from process $j$ to process $k \quad(j=1,2, k \neq j)$

$\mathrm{M}_{\mathrm{k}_{\mathrm{i}}}$ :messages from user $k$ to process $k$

$\mathrm{O}_{\mathrm{k}}$ : output actions of $k$-th process

$\mathrm{O}_{\mathrm{k}}=\left\{p ! m_{\mathrm{o}} \mid p \in \mathrm{SAP}_{\mathrm{k}}, m_{0} \in \mathrm{M}_{\mathrm{kj}} \cup \mathrm{M}_{\mathrm{k}_{0}}\right\}$

$\mathrm{M}_{\mathrm{k}_{0}}$ :messages from process $k$ to user $k$

$\omega_{\mathrm{k}}$ : output function of $k$-th process $\quad \mathrm{Q}_{\mathrm{k}} \times \mathrm{I}_{\mathrm{k}} \rightarrow \mathrm{O}_{\mathrm{k}}$ 
$\delta_{\mathrm{k}}$ : transition function of $k$-th process $\mathrm{Q}_{\mathrm{k}} \times \mathrm{I}_{\mathrm{k}} \rightarrow \mathrm{Q}_{\mathrm{k}}$

$q_{\mathrm{k} 0}:$ initial state of $k$-th process

In this definition, processes denotes tested protocol entities. "?"and“!" represent that these actions are input actions and output actions respectively. In addition, $\omega_{\mathrm{k}}\left(q, a_{\mathrm{i}}\right)=a_{0}$ and $\delta_{\mathrm{k}}\left(q, a_{\mathrm{i}}\right)=q$ ' means the transition that "under state $q$, when $a_{\mathrm{i}}$ is input, $a_{0}$ is output and state is changed into $q$," and is represented by " $q-a_{\mathrm{i}} / a_{0} \rightarrow \mathrm{q}$ ",

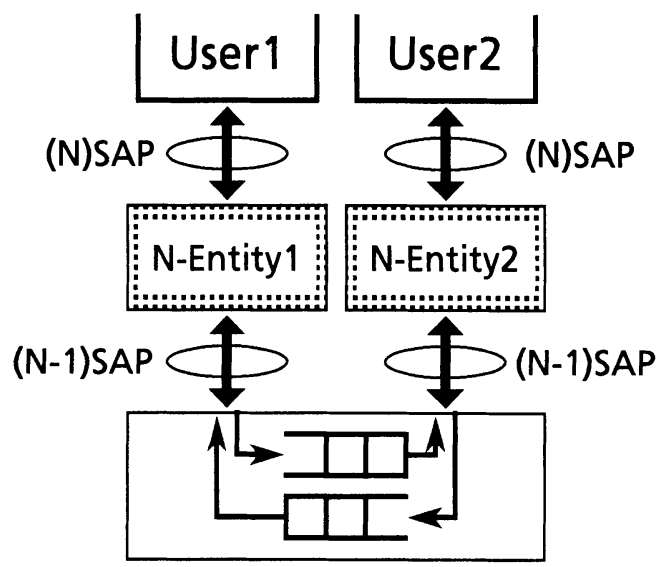

Fig. 1 Model of environment for system under test.

The environment of the interoperability testing is modeled as shown in Figure 2. The two protocol entities, (N)-Entity 1 and (N)-Entity 2, which are the tested systems, are called IUT(Implementation Under Test) 1 and IUT2 in this model. IUTs exchange test events(abstract service primitives) with the upper and lower testers via the upper and lower PCOs (Points of Control and Observation). The two lower testers communicate with each other via the $(\mathrm{N}-1)$ service provider. Hereinafter, the upper tester of IUTk is called UTk, the lower tester is called LTk, and the PCO between IUTk and UTk is called Uk, and the PCO between LTk and IUTk is called Lk.

The test sequences of the interoperability testing describe in what order the testers exchange test events in the four PCOs, U1, U2, L1 and L2.

\section{Formal Methods for Conformance Test Sequence Generation}

In this section, the concept behind the methods for identifying the states of FSM necessary for generating test sequences are presented and the existing methods for generating conformance test sequences are discussed.

\subsection{FSM state identification method}

While testing, in general, no one can know directly the (internal) state of the tested implementation. However, the state can be known indirectly from the output sequences obtained by adding certain input sequences, on the basis of the assumption that the specification definition is complete and minimal and that the state number of a implementation coincides with the number of states determined by specifications. As input sequences for identifying this state, W set[3], DS sequence[4], 

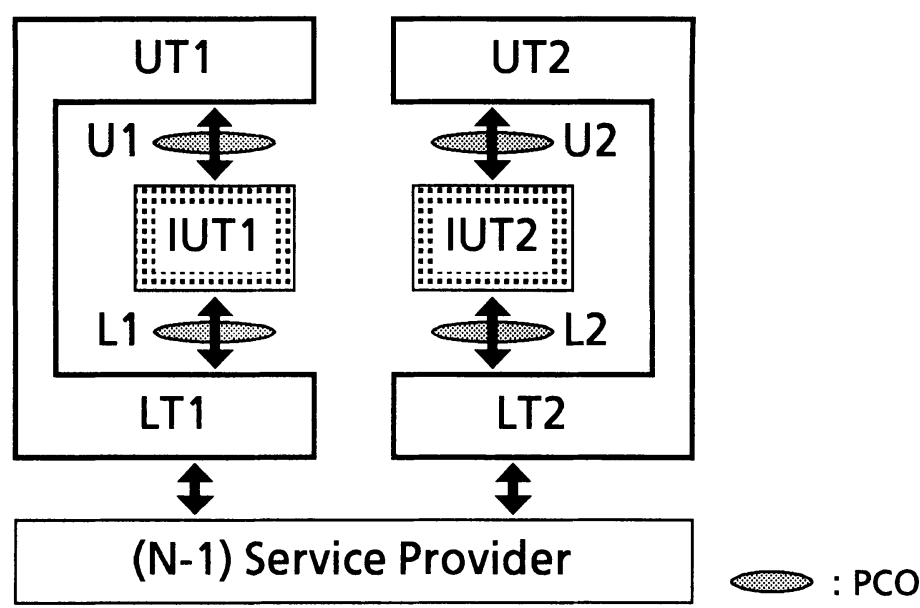

Fig. 2 Model of environment for interoperability testing.

and UIO sequence[5] are known. W set is a set of sequences composed of more than one input and identifies the state by adding all sequences that are elements of this W set with respect to each state. DS sequence is a sequence related to FSM and identify the states by being added to all states in the FSM. UIO sequence is a sequence related to each state of a FSM and identify the state by being added to each state respectively. The existence of $\mathrm{W}$ set is guaranteed[3]. Tere are some cases in which some states does not have UIO sequences, but we can use W set as substitution for UIO sequences with respect to these statets[5]. DS sequence does not exist in some cases.

For example, the UIO sequence for FSM of process 1 and process 2 constituting the protocol of Figure 3 becomes as shown in Table 1. If the output L1!m is obtained by adding the input $U 1$ ? $\mathrm{m}$ with respect to process 1 , we can know that the state is 1 before adding this input.

\subsection{Conformance test sequence generation methods}

FSM based Conformance test sequence generation methods are presented in References [2]-[5]3, among which Reference [2] presents a method by tracing a FSM from the initial state without knowing the (other) state. We call methods like this as trace methods. In addition, although References [3]-[5] differ in that the W set, DS sequence, and UIO sequence are used as methods of identifying each state of FSM, basically they present methods for generating test sequences that identify the states that perform the three steps in the basic test procedure(BTP) for all transitions. We call methods of this type as state oriented methods.

\section{[Basic testing procedure (BTP)]}

For each transition $\mathrm{sj}_{\mathrm{j}}-\alpha_{\mathrm{i}} / \alpha_{\mathrm{o}} \rightarrow \mathrm{s}_{\mathrm{k}}$,

(Step 1) Put the implementation to state $\mathrm{sj}$.

(Step 2) Give an input $\alpha_{\mathrm{i}}$ to the implementation, and observe the output $\alpha_{0}$.

(Step 3) Confirm that the implementation is in state $\mathrm{s}_{\mathrm{k}}$.

3 These methods assume that FSMs have reset sequences which bring the machines to the initial state. In this paper we also assume the existance of reset sequences. 


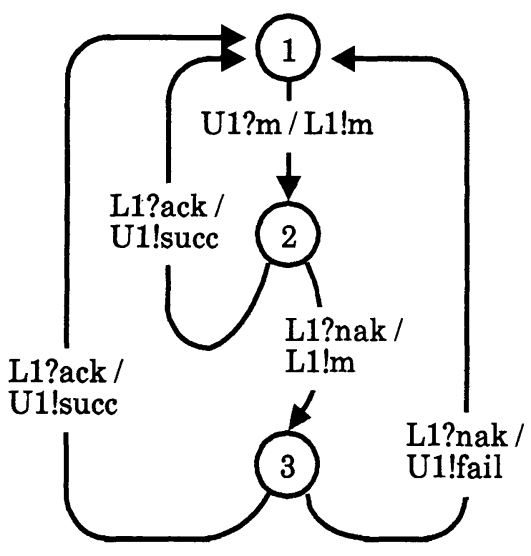

Process 1

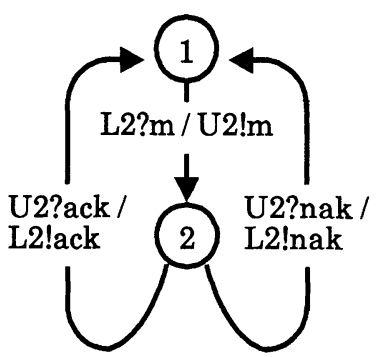

Process 2

Fig.3 An Example Protocol $P_{\text {ex. }}$

\begin{tabular}{|c|c|c|c|}
\hline \multicolumn{2}{|c|}{ process 1 } & \multicolumn{2}{c|}{ process 2 } \\
\hline States & UIO & States & UIO \\
\hline 1 & U1?m/L1!m & 1 & L2?m / U2!m \\
\hline 2 & L1?nak /L1!m & 2 & U2?ack / L2!ack \\
\hline 3 & L1?nak / U1!fail & - & - \\
\hline
\end{tabular}

Table 1 UlO sequences.

Regarding these methods, it has been known that a part of errors of the output/transition function that cannot be detected by the test sequences generated by trace method[6] can be detected by state oriented methods. The error detection coverages of latter methods are larger than that of former one.

\section{Interoperability Test Sequence Generation methods}

\subsection{Introduction of the system state graph}

When the protocol is defined as two FSMs as in Definition 1, two test sequences can be obtained by applying the existing conformance test sequence generation methods to each process. In interoperability testing, however, the sequential relationships between each action in the two test sequences obtained independently are important. For example, when the action sequences in the two test sequences are $a_{1} b_{1} c_{1}$ and $d_{2} e_{2} f_{2}$, since information on sequential relationships between $b_{1}$ and $d_{2}$, $e_{2}, f_{2}$ is lacking, although it is known that $b_{1}$ occurs after $a_{1}$ and before $c_{1}$, interoperability testing cannot be performed by these two sequences. Such a problem is called a scheduling problem in interoperability testing here.

In order to resolve this scheduling problem, the system state graph (SSG: System State Graph) showing the behaviours of the whole system is introduced. SSG is a graph with the system states as nodes that can be obtained by synthesizing the states 
of the two processes and the states of the channels between these processes. The definition of SSG is given below. Each channel is assumed to be FIFO.

[Definition 2] System state $s=\left\langle q_{1}, q_{2}, c_{12}, c_{21}\right\rangle$ where

$q_{k}$ : state of $k$-th process, $q_{k} \in Q_{k}$

$c_{\mathrm{ij}}$ : channel contents from $\mathrm{i}$-th process to $\mathrm{j}$-th process, $\mathrm{c}_{\mathrm{ij}} \in \mathrm{M}_{\mathrm{ij}}{ }^{*}(i, j=1,2, i \neq j)$

[Definition 3] SSG G $=\left\langle\mathrm{S}, \mathrm{I}, \mathrm{O}, \omega, \delta, \mathrm{s}_{0}\right\rangle$

where

S: set of system states

I: set of input actions

0 : set of output actions

$\omega:$ output function $\mathrm{S} \times \mathrm{I} \rightarrow 0$

$\delta:$ transition function $\mathrm{S} \times \mathrm{I} \rightarrow \mathrm{S}$

so: initial system state

Where, $\omega\left(\mathbf{s}, \alpha_{\mathrm{i}}\right)=\alpha_{0}$ and $\delta\left(\mathbf{s}, \alpha_{\mathrm{i}}\right)=\mathrm{s}^{\prime}$ means the transition that if $\alpha_{\mathrm{i}}$ is input in system state $\mathbf{s}, \alpha_{0}$ is output and transition to system state s' occurs, and is represented by s$\alpha_{\mathrm{i}} / \alpha_{0} \rightarrow \mathrm{s}^{\prime}$.

Next, the rules for generating a SSG from protocol $P$ are given as follows :

[SSG generation rules] Given that protocol $\mathrm{P}=\left\langle<\mathrm{Q}_{1}, \mathrm{I}_{1}, \mathrm{O}_{1}, \omega_{1}, \delta_{1}, \mathrm{q}_{10}\right\rangle,<\mathrm{Q}_{2}$, $\mathrm{I}_{2}, \mathrm{O}_{2}, \omega_{2}, \delta_{2}, \mathrm{q}_{20}>>, \mathrm{SSG} \mathrm{G}=<\mathrm{S}, \mathrm{I}, \mathrm{O}, \omega, \delta, \mathrm{s}_{0}>$ composed of the system state set $\mathrm{S}$, output function $\omega$, transition function $\delta$, and initial system state $\mathrm{s}_{0}$, that is inferred from $\mathrm{I}=\mathrm{I}_{1} \cup \mathrm{I}_{2}, \mathrm{O}=\mathrm{O}_{1} \cup \mathrm{O}_{2}$, the next axiom $\mathrm{A} 1$ and inference rules $\mathrm{I} 1 \sim \mathrm{I} 8$ determines SSG with respect to $\mathrm{P}$. Where $E$ represents that channel content is empty. Also, append $\left(c_{\mathrm{kj}}, m\right)$, top $\left(c_{\mathrm{kj}}\right)$, remain $\left(c_{\mathrm{kj}}\right)$ are determined as follows:

append $\left(c_{\mathrm{kj}}, m\right)$ : channel of the state in which $m$ is added to the end of channel $c_{\mathrm{kj}}$.

$t o p\left(c_{\mathrm{kj}}\right)$ : contents of the beginning of channel $c_{\mathrm{kj}}$. It is not defined in case channel $c_{\mathrm{kj}}$ is empty.

remain $\left(c_{\mathrm{kj}}\right)$ : channel of the state with the contents of the beginning of channel $c_{\mathrm{kj}}$

A1 (Initial system state)

eliminated. This is not defined in case channel $c_{\mathrm{kj}}$ is empty.

$s_{0}=\left\langle q_{10}, q_{20}, E, E>\in \mathrm{S}\right.$

I1 (Input and output on U1)

where, $s^{\prime}=\left\langle q_{1}^{\prime}, q_{2}, c_{12}, c_{21}\right\rangle$

$$
\frac{s=\left\langle q_{1}, q_{2}, c_{12}, c_{21}>\in \mathrm{S}, \quad q_{1}-\mathrm{U} 1 ? m_{1_{\mathrm{i}}} / \mathrm{U} 1 ! m_{1_{0}} \rightarrow q_{1}{ }^{\prime}\right.}{s-\mathrm{U} 1 ? m_{1_{\mathrm{i}}} / \mathrm{U} 1 ! m_{1_{0}} \rightarrow s^{\prime}}
$$

I2 (Input on U1 and output on L1)

$$
\frac{s=\left\langle q_{1}, q_{2}, c_{12}, c_{21}>\in \mathrm{S}, \quad q_{1}-\mathrm{U} 1 ? m_{1} / \mathrm{L} 1 ! m_{12} \rightarrow q_{1}^{\prime}\right.}{s-\mathrm{U} 1 m_{1_{\mathrm{i}}} / \mathrm{L} 1 ! m_{12} \rightarrow s^{\prime}}
$$

where, $s^{\prime}=\left\langle q_{1}{ }^{\prime}, q_{2}\right.$, append $\left.\left(c_{12}, m_{12}\right), c_{21}\right\rangle$ 
I3 (Input on L1 and output on U1)

where, $s^{\prime}=\left\langle q_{1}{ }^{\prime}, q_{2}, c_{12}\right.$, remain $\left.\left(c_{21}\right)\right\rangle$

$$
\begin{aligned}
& s=\left\langle q_{1}, q_{2}, c_{12}, c_{21}\right\rangle \in \mathrm{S}, \quad \operatorname{top}\left(c_{21}\right)=m_{21}, q_{1}-\mathrm{L} 1 ? m_{21} / \mathrm{U} 1 ! m_{1_{0}} \rightarrow q_{1}{ }^{\prime} \\
& s-\mathrm{L} 1 ? m_{21} / \mathrm{U} 1 ! m_{1} \rightarrow s^{\prime}
\end{aligned}
$$

I4 (Input and output on L1)

$$
\frac{s=\left\langle q_{1}, q_{2}, c_{12}, c_{21}>\in \mathrm{S}, \quad \text { top }\left(c_{21}\right)=m_{21}, q_{1}-\mathrm{L} 1 m_{21} / \mathrm{L} 1 ! m_{12} \rightarrow q_{1}{ }^{\prime}\right.}{s-\mathrm{L} 1 ? m_{21} / \mathrm{L} 1 ! m_{12} \rightarrow s^{\prime}}
$$

where, $s^{\prime}=\left\langle q_{1}, q_{2}\right.$, append $\left(c_{12}, m_{12}\right)$, remain $\left.\left(c_{21}\right)\right\rangle$

I5 (Input and output on U2)

where, $s^{\prime}=\left\langle q_{1}, q_{2}^{\prime}, c_{12}, c_{21}\right\rangle$

$$
\frac{s=\left\langle q_{1}, q_{2}, c_{12}, c_{21}>\in \mathrm{S}, q_{2}-\mathrm{U} 2 ? m_{2_{\mathrm{i}}} / \mathrm{U} 2 ! m_{2_{0}} \rightarrow q_{2}{ }^{\prime}\right.}{s-\mathrm{U} 2 ? m_{2_{\mathrm{i}}} / \mathrm{U} 2 ! m_{2_{0}} \rightarrow s^{\prime}}
$$

I6 (Input on U2 and output on L2)

$$
\frac{s=\left\langle q_{1}, q_{2}, c_{12}, c_{21}>\in \mathrm{S}, \quad q_{2}-\mathrm{U} 2 ? m_{2_{\mathrm{i}}} / \mathrm{L} 2 ! m_{21} \rightarrow q_{2}\right.}{s-\mathrm{U} 2 ? m_{2_{\mathrm{i}}} / \mathrm{L} 2 ! m_{21} \rightarrow s^{\prime}}
$$

where, $s^{\prime}=\left\langle q_{1}, q_{2}, c_{12}\right.$, append $\left.\left(c_{21}, m_{21}\right)\right\rangle$

I7 (Input on L2 and output on U2)

where, $s^{\prime}=\left\langle q_{1}, q_{2}\right.$, remain $\left(c_{12}\right), c_{21}>$

$$
\frac{s=\left\langle q_{1}, q_{2}, c_{12}, c_{21}>\epsilon \mathrm{S}, \quad \text { top }\left(c_{12}\right)=m_{12}, q_{2}-\mathrm{L} 2 ? m_{12} / \mathrm{U} 2 ! m_{2_{0}} \rightarrow q q_{2}\right.}{s-\mathrm{L} 2 ? m_{12} / \mathrm{U} 2 ! m_{2_{0}} \rightarrow s^{\prime}}
$$

$\underline{\text { I8 }}$ (Input and output on L2)

$$
\begin{aligned}
& \frac{s=\left\langle q_{1}, q_{2}, c_{12}, c_{21}>\in \mathrm{S}, \quad \text { top }\left(c_{12}\right)=m_{12}, q_{2}-\mathrm{L} 2 ? m_{12} / \mathrm{L} 2 ! m_{21} \rightarrow q_{2}\right.}{s-\mathrm{L} 2 ? m_{12} / \mathrm{L} 2 ! m_{21} \rightarrow s^{\prime}} \\
& \text { where, } s^{\prime}=\left\langle q_{1}, q_{2}, \text { remain }\left(c_{12}\right), \text { append }\left(c_{21}, m_{21}\right)>\right.
\end{aligned}
$$

In some protocol specifications, there are cases to which applications of these rules do not end in a finite number of times. For example, in process $\mathrm{p}_{1}$, if a self-loop transition labelled by input on $\mathrm{U} 1$ and output on $\mathrm{L} 1$ at any state is defined, rule I2 can be applied an infinite number of times. However, this problem is in general impossible to solve, so it is not taken as an object of discussion here. In this study, only the cases for which applications of these rules end in a finite number of times are considered. Thus, SSG is treated as a finite graph below.

As shown in Section 4.2 below, by tracing SSG test sequences are obtained as sequences of actions of the whole system. In such test sequences, the sequential 
relationships between actions of the whole system are clearly indicated. Thus, the scheduling problem is resolved.

\subsection{Interoperability test sequence generation method}

In this section, the method of generating interoperability test sequences using SSG shown in Section 4.1 is presented. In Subsection 4.2.1, first, existing method[10] is introduced briefly and problems associated with it are discussed. Next, a method of applying existing state based conformance test sequence generation methods[3]-[5] to SSG directly, named "simple method", is discussed. This method is effective for testing that does not consider independency of the processes. In Subsection 4.2.2, then limitations of this simple method from the point of view of more strict testing are discussed. To overcome these limitations, a new interoperability test sequence generation method, named "IP method", considering the independency of the processes is proposed.

\subsubsection{Simple method}

In Reference [10], the method of generating test sequences by using the reachability graph, which is similar to SSG discussed in the preceding section and tracing the reachability graph is presented using a simple example. This method is called the trace method here. For example, as shown later, SSG $\mathrm{G}_{\mathrm{ex}}$ corresponding to the protocol $P_{e x}$ of Figure 3 becomes as shown in Figure 4 . With respect to this $G_{e x}$, one of the test sequences obtained by the trace method becomes

$\mathrm{U} 1$ ? $\mathrm{m}$; L2? $\mathrm{m}$; U2?ack ; L1?ack.

However, as with the case of the conformance test sequence generation methods, the trace method that does not identify states has a problem of not having the capability to detect a part of errors of the output/transition function. Here, a method identifying states and resolving the above problem is discussed below.

SSG is itself a kind of FSM, and a minimal one is obtained by applying the algorithm for minimizing FSM. In addition, this SSG can be regarded as completely defined by assuming self-loop transitions with an undefined input and an empty output. It can be considered as strongly connected by assuming a reset sequence for returning to the initial state for each state. The above facts indicate that SSG derived above satisfies the limiting conditions common in the PW method, DS method, UI method, which are existing conformance test sequence generation methods based on identification of states (the DS method requires that a DS sequence exists). Thus, it is naturally considered that these methods can be applied to obtain test sequences for this SSG. This is called the simple method below. Since by the simple method, all the errors of the output function and the transition function when SSG is taken as FSM can be detected, the method is effective for testing that does not consider the independency of the processes.

\subsubsection{IP method: A new Method Considering the Independency of Processes}

The various methods given above for generating conformance test sequences have testing single processes as their goal. On the other hand, in interoperability testing we test essentially a number of independent processes. Thus, as shown below, the simple method, which applies these methods as they are to SSG, is insufficient for generating more strict and effective test sequences considering the independency of the processes. For example, regarding the protocol $\mathrm{P}_{\mathrm{ex}}$ of Figure 3, generating test 


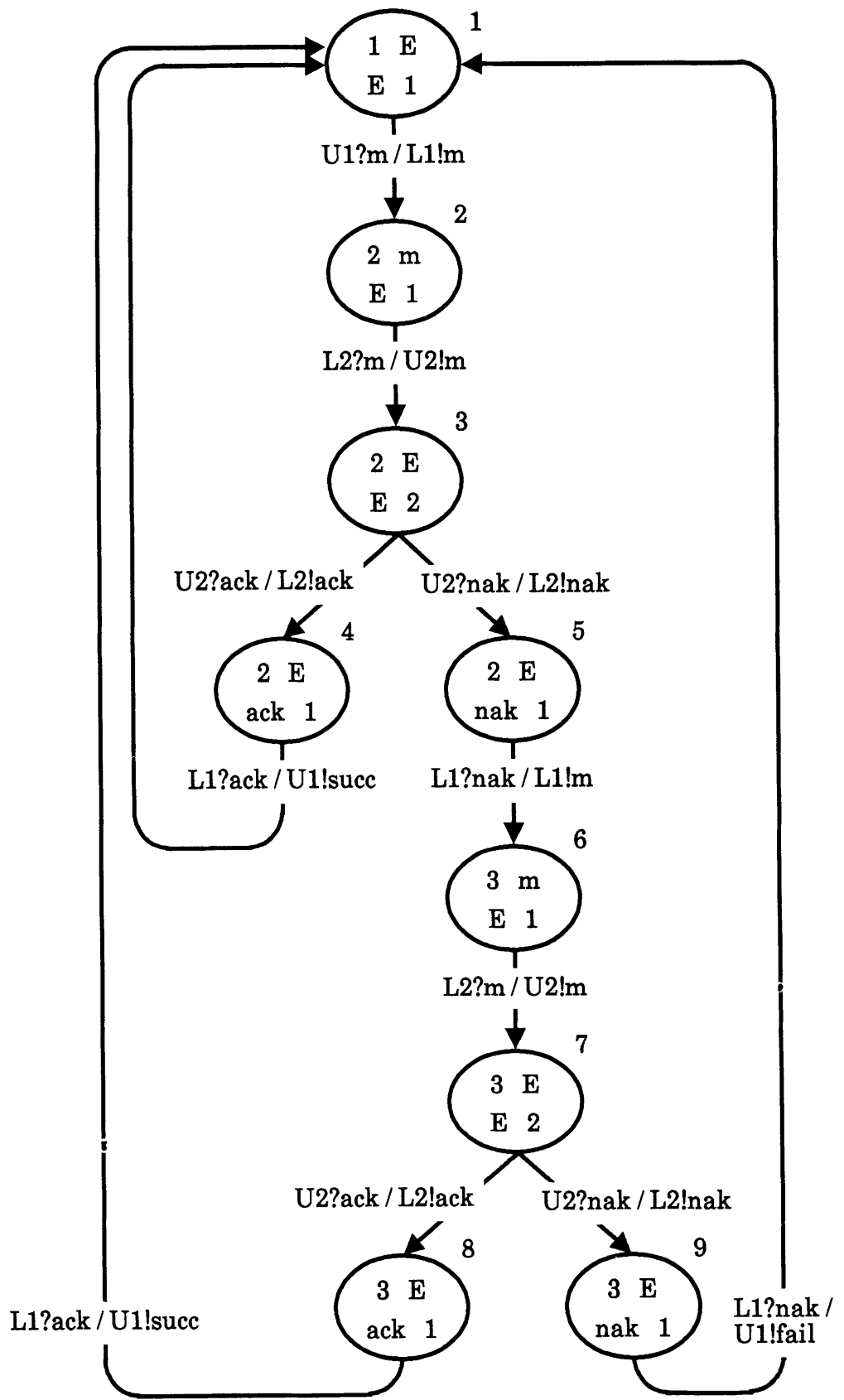

Fig.4 SSG Gex. 
sequences using the UI method may be considered. In this case, the corresponding SSG becomes $\mathrm{G}_{\mathrm{ex}}$ as shown in Figure 4. If $\mathrm{G}_{\mathrm{ex}}$ is regarded as a FSM here, this contains redundant states. Here, it is necessary to minimize by the FSM minimization algorithm. SSG G'ex (Figure 5) obtained by minimization is completely defined, strongly connected under the assumption discussed above. For this G' $_{\text {ex }}$ UIO sequences can be obtained. Test sequences are generated based on the steps of BTP steps 1-3 for each transition of $\mathrm{G}_{\text {'ex }}$ using these sequences. For example, for the transition "3-U 2?ack/ L2!ack $\rightarrow$," this sequence becomes "r; U1?m; U2?m; U2?ack; L1?ack." Here, $r$ is a reset sequence. At this time, the sequence corresponding to Step 3, or the sequence confirming the system state 4 is L1?ack. Since, in interoperability testing, process 1 and process 2 operate essentially independently, it is necessary to confirm the state of each process in order to confirm the "state" under the test environment. In this case, it is necessary to confirm state 2 of process 1 and state 1 of process 2 corresponding to system state 4 . Although by the sequence obtained above, the input of L1?ack is given with respect to process 1 , the fact that this process is in state 2 cannot be confirmed. Now, the output obtained by giving this input to process 1 is the same for state 2 and state 3 , so the two states cannot be identified by this input. In addition, since an input is not given to process 2 by the sequence obtained above, the state of this process cannot be confirmed. As can be understood from these facts, confirming the state preceding a transition in step 3 of BTP is insufficient by the simple method.

Thus, the simple method shown in 4.2.1 is insufficient for generating strict test sequences considering the independency of the processes. This is due to the fact that a SSG is treated as a FSM by the simple method so the individual states of each process constituting the system states are not considered, while they have to be considered because it is necessary to treat essentially independent processes in interoperability testing.

In view of the above facts, a new interoperability test sequences generation method, named "IP method" is proposed as follows. By the IP method, sequences performing the three steps of BTP are generated on SSG. This method has a special feature in that not only confirming sequences (UIO sequences in the UI method, etc.) applied in confirming the states of individual processes constituting system states is conformed, but also considering the independency of processes, which is a characteristic of interoperability testing. Therefore, this method is defined as follows.

\section{[Interoperability test sequence generation method: IP method]}

(Phase 1) SSG G $=<\mathrm{S}, \mathrm{I}, \mathrm{O}, \omega, \delta, \mathrm{s}_{0}>$ is generated from protocol $\mathrm{P}=<<\mathrm{Q}_{1}, \mathrm{I}_{1}, \mathrm{~T}_{1}$, $\omega_{1}, \delta_{1}, \mathrm{q}_{10}>,\left\langle\mathrm{Q}_{2}, \mathrm{I}_{2}, \mathrm{~T}_{2}, \omega_{2}, \delta_{2}, \mathrm{q}_{20}>>\right.$ in accordance with the SSG generation rules.

(Phase 2) For process 1 , the sequence $u\left(q_{1 k}\right)$ identifying $q_{1 k} \in Q_{1}$ uniquely is obtained. $u\left(q_{2 k}\right)$ is obtained similarly for process 2 .

(Phase 3) Constructs sequences corresponding to steps 1-3 of BTP for each transition $\mathrm{s}_{\mathrm{i}}-\alpha_{\mathrm{in}} / \alpha_{\text {out }} \rightarrow \mathrm{sj}_{\mathrm{j}}$ (with $\left.\left.\mathrm{s}_{\mathrm{i}}=<\mathrm{q}_{1}, \mathrm{q}_{2}, \mathrm{c}_{12}, \mathrm{c}_{21}\right\rangle, \mathrm{sj}_{\mathrm{j}}=\left\langle\mathrm{q}_{1}^{\prime}, \mathrm{q}_{2}{ }_{2}, \mathrm{c}_{12}^{\prime}, \mathrm{c}_{21}\right\rangle\right)$, which is $\omega\left(\mathrm{s}_{\mathrm{i}}\right.$, $\left.\alpha_{\text {in }}\right)=\alpha_{\text {out }}, \delta\left(\mathrm{si}_{\mathrm{i}}, \alpha_{\text {in }}\right)=\mathrm{sj}_{\mathrm{j}}$ on SSG, as shown in (1) (3) below:

(1) $r ; x\left(s_{0}, s_{\mathrm{i}}\right)$; (2) $a_{\mathrm{in}}$; (3) $u\left(q_{1}^{\prime}\right) ; u\left(q_{2}{ }^{\prime}\right)$

Where, $r$ represents the reset sequence, $x\left(\mathrm{~s}_{0}, \mathrm{~s}_{\mathrm{i}}\right)$ the sequence of the input that is 


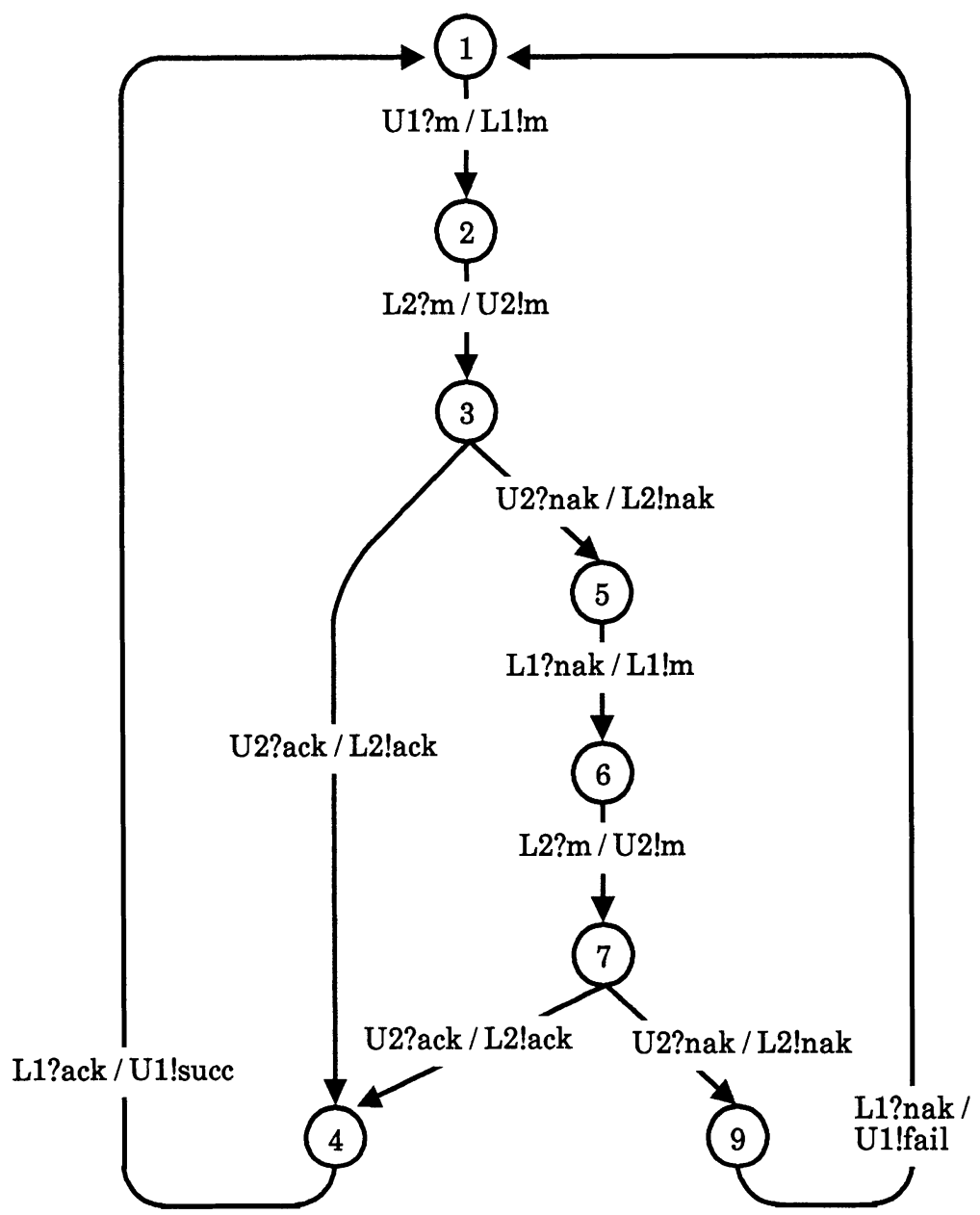

Fig.5 Reduced SSG $G_{e x}$.

transitional from the initial system state $\mathrm{s}_{0}$ to system state $\mathrm{s}_{\mathrm{i}}$. In addition, “; " represents the concatenation of the sequence.

First, in phase 1, the executable protocol behavior of the whole system is obtained as a preparation for testing, in order to know the scope of testing. Next, in phase 2, sequences identifying the states of each process appearing on SSG are obtained by existing methods. These are used in the next phase. Finally, in phase 3 , sequences checking whether each process realizes an appropriate transition are generated on the basis of information of phase 1 and phase 2 .

\subsection{Application and Evaluation of IP Method}

An example of applying the IP method to actual specifications is presented. The protocol specification $\mathrm{P}_{\mathrm{ex}}$ shown in Figure 3 is considered. This specification example focuses for example on the transition 2-U2?ack / L2!ack $\rightarrow 1$ of process 2. 
For this transition, the transition $<2,2, \mathrm{E}, \mathrm{E}>-\mathrm{U} 2$ ?ack / L2!ack $\rightarrow<2,1, \mathrm{E}$, ack $>$ is inferred by applying SSG generation rule I6 shown in 4.1. Thus, by the specifications of Figure 3, SSG G $_{\text {ex }}$ in Figure 4 is obtained (phase 1) by applying the SSG generation rules. In this figure, an oval represents a system state $s=<q_{1}, q_{2}$, $c_{12}, c_{21}>$, and the upper left, lower right, upper right and lower left represent $q_{1}, q_{2}$, $c_{12}$ and $c_{21}$ respectively. Next, if for example, a UIO sequence is obtained as state identifying sequence for FSM of each process of Figure 3, Table 1 results (phase 2). In $\mathrm{G}_{\mathrm{ex}}$, the sequence for the transition $<2,2, \mathrm{E}, \mathrm{E}>-\mathrm{U} 2$ ?ack / L2!ack $\rightarrow<2,1, \mathrm{E}$, ack $>$ can be obtained as

(1) $r$; U1? $m$; L2? $m$; (2) U2?ack; (3) L1? nak; L2?m

where, part of "L1?nak" is the UIO sequence identifying state 2 of process 1 , and part of "L2?m" is the UIO sequence identifying state 1 of process 2 . Thus, the test sequences obtained for each transition of $\mathrm{G}_{\mathrm{ex}}$ of Figure 4 become as shown in Figure 6 (phase 3). The underlined parts of this figure correspond to parts of (2). In these test sequences, "U1?m" for example represents the action that "test event $m$ is input on PCO U1." In addition, since the corresponding output sequences are obtained from protocol specifications when the input sequences from the initial state are given, output actions are omitted and test sequences are expressed by only input actions.

\begin{tabular}{|c|c|}
\hline transition & test sequence \\
\hline $1-\mathrm{U} 1 ? \mathrm{~m} / \mathrm{L} 1 \mathrm{~m} \rightarrow 2$ & $\mathrm{r} ; \underline{\mathrm{U} 1 ? \mathrm{~m}}$; L1?nak; L2?m \\
\hline $2-\mathrm{L} 2 ? \mathrm{~m} / \mathrm{U} 2 ! \mathrm{m} \rightarrow 3$ & 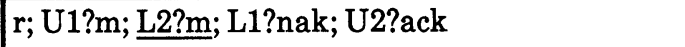 \\
\hline 3-U2?ack / L2!ack $\rightarrow 4$ & $\mathrm{r} ; \mathrm{U1}$ ?m; L2?m; U2?ack; L1?nak; L2?m \\
\hline 3-U2?nak / L2!nak $\rightarrow 5$ & r; U1?m; L2?m; U2?nak; L1?nak; L2?m \\
\hline 4-L1?ack / U1!succ $\rightarrow 1$ & $\mathrm{r} ; \mathrm{U1}$ ?m; L2?m; U2?ack; L1?ack; U1?m; L2?m \\
\hline 5-L1?nak / L1!m $\rightarrow 6$ & r; U1?m; L2?m; U2?nak; L1?nak; L1?nak; L2?m \\
\hline $6-\mathrm{L} 2 ? \mathrm{~m} / \mathrm{U} 2 ! \mathrm{m} \rightarrow 7$ & $\begin{array}{l}\text { r; U1?m; L2?m; U2?nak; L1?nak; L2?m; L1?nak; } \\
\text { U2?ack }\end{array}$ \\
\hline 7-U2?ack / L2!ack $\rightarrow 8$ & $\begin{array}{l}\text { r; U1?m; L2?m; U2?nak; L1?nak; L2?m; U2?ack; } \\
\text { L1?ack; U1?m; L2?m }\end{array}$ \\
\hline 8-L1?ack / U1!succ $\rightarrow 1$ & $\begin{array}{l}\text { r; U1?m; L2?m; U2?nak; L1?nak; L2?m; U2?ack; } \\
\text { L1?ack; U1?m; L2?m }\end{array}$ \\
\hline 7-U2?nak / L2!nak $\rightarrow 9$ & $\begin{array}{l}\text { r; U1?m; L2?m; U2?nak; L1?nak; L2?m; U2?nak; } \\
\text { L1?nak; L2?m }\end{array}$ \\
\hline 9-L1?nak / U1!fail $\rightarrow 1$ & $\begin{array}{l}\text { r; U1?m; L2?m; U2?nak; L1?nak; L2?m; U2?nak; } \\
\text { L1?nak; U1?m; L2?m }\end{array}$ \\
\hline
\end{tabular}

Fig.6 Test Sequences.

Using the test sequences obtained thus, interoperability testing can be performed in the environment of Figure 2 as follows. In the order of appearance of each action "P?asp" in a test sequence, by any of UT 1, UT 2, LT 1, LT 2, which are testers corresponding to PCO $\mathrm{P}$, which is any of $\mathrm{U} 1, \mathrm{U} 2, \mathrm{~L} 1, \mathrm{~L} 2$, test event asp is given as stimulus and the response of the output is observed. A tester confirms the fact that this output action coincides with the output action obtained from protocol 
specifications. Only when the above is completed without abnormality for all actions in all test sequences, the "correctness" of implementation is guaranteed. The above is considered to be determined separately for methods of realization of synchronization between each tester and communications between the two lower testers LT 1 and LT 2, which are called the testing management protocol.

The three steps of BTP are performed for each transition in SSG by the IP method. By this fact, except for very limited cases[7] all errors of the transition function and the output function of SSG can be detected as in the discussion of conformance testing[6]. The cost associated with the amount of calculations involved and sequence lengths etc. of this method depends on the input number and state number of SSG. The problems of cost reduction and quantitative analysis etc. are not objects of this study.

As discussed above, by the IP method which is the interoperability test sequence generation method proposed by this study, the state transition corresponding to step 3 of BTP can be confirmed strictly by considering the independency of each process, which is an essential property of interoperability testing. By this fact, more strict and effective test sequences to the level of processes that cannot to be considered by the simple method can be generated.

Next, an evaluation for IP method is given using an example protocol. To investigate the error detection coverage of IP method comparing with the trace method and the simple saethod, we verify that each method can detect errors of output/ transition function of FSMs.

As mentioned above, test sequences obtained from the protocol in Figure 3 by the trace method are

1) U1?m; L2?m; U2?ack ; L1?ack,

2) U1?m; L2?m; U2?nak ; L1?nak ; L2?m ; U2?ack ; L1?ack,

3) U1?m; L2?m ; U2?nak ; L1?nak ; L2?m ; U2?nak ; L1?nak.

In transitions

$1-\mathrm{U} 1$ ? $\mathrm{m} / \mathrm{L} 1 \mathrm{~m} \rightarrow 2,2-\mathrm{L} 2$ ? $\mathrm{m} / \mathrm{U} 2$ ! $\mathrm{m} \rightarrow 3,3-\mathrm{U} 2$ ?ack / L2!ack $\rightarrow 4$ and 4 -L1?ack / $\mathrm{U} 1$ !succ $\rightarrow 1$ (denoted below as $1 \rightarrow 2,2 \rightarrow 3,3 \rightarrow 4$ and $4 \rightarrow 1$, respectively) which construct sequence 1 ), we consider $3 \rightarrow 4$ and $4 \rightarrow 1$. If it is verified that $1 \rightarrow 2$ and $2 \rightarrow$ 3 are correct, step 1 of BTP about $3 \rightarrow 4$ is executed by input actions "U1?m; L2?m". Also step 2 is executed by input action "U2?ack". So the output function of $3 \rightarrow 4$ is verified. On the other hand, step 3 of BTP about $3 \rightarrow 4$ is not executed because input actions "L1?nak ; L2?m" which are needed to verify the state 4 are not included in sequence 1). So the transition function of $3 \rightarrow 4$ is not verified. In addition, step 1 of BTP about $4 \rightarrow 1$ is not executed correctly because the transition function of $3 \rightarrow 4$ is not verified. It leads that both the output function and the transition function of $3 \rightarrow$ 4 are not verified. Table 2 denotes the result of check that steps $1 \sim 3$ of each function are verified. This is the error detection coverage of the trace method about the example protocol. In the same way we obtain the results of the error detection coverage of the simple method and IP method about the example protocol shown in Table 3 and Table 4, respectively. Table 5 shows the comparison of the error detection coverages about three methods. From this table, following results are obtained.

a) Part of, but not all, errors that can not be detected by test sequences generated by 
the trace method can be detected by the test sequences generated by the simple method,

b) All errors that can not be detected by test sequences generated by the trace method or the simple method can be detected by the test sequences generated by the IP method.

From these points, we can conclude that the IP method can improve the error detection coverage of interoperability testing.

\begin{tabular}{|c|c|c|c|c|c|c|c|c|c|c|}
\hline \multirow{2}{*}{$\begin{array}{c}\text { transi- } \\
\text { tion }\end{array}$} & \multirow{2}{*}{ step } & \multirow{2}{*}{$\begin{array}{c}\text { step } \\
2\end{array}$} & \multicolumn{6}{|c|}{ step3 } & \multirow[b]{2}{*}{$\omega$} & \multirow[b]{2}{*}{$\delta$} \\
\hline & & & state & $\frac{\text { process } 1}{U 10}$ & check & state & $\begin{array}{c}\text { process } 2 \\
\text { UIO }\end{array}$ & check ] & & \\
\hline $1 \rightarrow 2$ & 0 & 0 & 2 & L1?nak & 0 & 1 & $L 2 ? m$ & $\bar{O}$ & 0 & 0 \\
\hline $2 \rightarrow 3$ & 0 & 0 & 2 & L1?nak & 0 & 2 & U2?nak & 0 & 0 & 0 \\
\hline $3 \rightarrow 4$ & 0 & 0 & 2 & L1?nak & $x$ & 1 & $L 2 ? m$ & $x$ & 0 & $x$ \\
\hline $3 \rightarrow 5$ & 0 & 0 & 2 & L1?nak & 0 & 1 & L2?m & 0 & 0 & 0 \\
\hline $4 \rightarrow 1$ & $x$ & 0 & 1 & U1?m & $x$ & 1 & $L 2 ? m$ & $x$ & $x$ & $x$ \\
\hline $5 \rightarrow 6$ & 0 & 0 & 3 & L1?nak & 0 & 1 & $L 2 ? m$ & 0 & 0 & 0 \\
\hline $6 \rightarrow 7$ & 0 & 0 & 3 & L1?nak & 0 & 2 & U2?nak & 0 & 0 & 0 \\
\hline $7 \rightarrow 8$ & 0 & 0 & 3 & L1?nak & $x$ & 1 & $L 2 ? m$ & $x$ & 0 & $x$ \\
\hline $8 \rightarrow 1$ & $x$ & 0 & 1 & U1?m & $x$ & 1 & $L 2 ? m$ & $x$ & $x$ & $x$ \\
\hline $7 \rightarrow 9$ & 0 & 0 & 3 & L1?nak & 0 & 1 & $L 2 ? m$ & $x$ & 0 & $\Delta$ \\
\hline $9 \rightarrow 1$ & $x$ & 0 & 1 & U1?m & $x$ & 1 & $L 2 ? m$ & $x$ & $x$ & $x$ \\
\hline
\end{tabular}

Table 2 Error detection coverage of trace method[10].

\begin{tabular}{|c|c|c|c|c|c|c|c|c|c|c|}
\hline \multirow{3}{*}{$\begin{array}{c}\text { transi- } \\
\text { tion }\end{array}$} & \multirow{3}{*}{$\begin{array}{c}\text { step } \\
1\end{array}$} & \multirow{3}{*}{$\begin{array}{c}\text { step } \\
2\end{array}$} & \multicolumn{6}{|c|}{ step3 } & \multirow{3}{*}{$\omega$} & \multirow{3}{*}{$\delta$} \\
\hline & & & \multicolumn{3}{|c|}{ process 1} & \multicolumn{3}{|c|}{ process 2} & & \\
\hline & & & state & UIO & check & state & UIO & check & & \\
\hline $1 \rightarrow 2$ & 0 & 0 & 2 & L1?nak & 0 & 1 & $L 2 ? m$ & 0 & 0 & 0 \\
\hline $2 \rightarrow 3$ & 0 & 0 & 2 & L1?nak & 0 & 2 & U2?nak & 0 & 0 & 0 \\
\hline $3 \rightarrow 4$ & 0 & 0 & 2 & L1?nak & $x$ & 1 & $L 2 ? m$ & $x$ & 0 & $x$ \\
\hline $3 \rightarrow 5$ & 0 & 0 & 2 & L1?nak & 0 & 1 & $L 2 ? m$ & 0 & $\mathrm{O}$ & 0 \\
\hline $4 \rightarrow 1$ & $x$ & 0 & 1 & U1?m & 0 & 1 & $L 2 ? m$ & $x$ & $x$ & $\Delta$ \\
\hline $5 \rightarrow 6$ & 0 & 0 & 3 & L1?nak & 0 & 1 & $L 2 ? m$ & 0 & 0 & 0 \\
\hline $6 \rightarrow 7$ & 0 & 0 & 3 & L1?nak & 0 & 2 & U2?nak & 0 & 0 & 0 \\
\hline $7 \rightarrow 4$ & 0 & 0 & 3 & L1?nak & $x$ & 1 & $L 2 ? m$ & $x$ & 0 & $x$ \\
\hline $7 \rightarrow 9$ & 0 & 0 & 3 & L1?nak & 0 & 1 & $L 2 ? m$ & $x$ & 0 & $\Delta$ \\
\hline $9 \rightarrow 1$ & $\bar{x}$ & 0 & 1 & U1?m & 0 & 1 & $L 2 ? m$ & $x$ & $x$ & $\Delta$ \\
\hline
\end{tabular}

Table 3 Error detection coverage of simple method.

\section{Conclusions}

In this study, a new method for generation of interoperability test sequences is proposed. In this method, test sequences are generated by constructing a system state graph showing the behaviours of the whole system and by identifying states on 


\begin{tabular}{|c|c|c|c|c|c|c|c|c|c|c|}
\hline \multirow{3}{*}{$\begin{array}{c}\text { transi- } \\
\text { tion }\end{array}$} & \multirow{3}{*}{$\begin{array}{c}\text { step } \\
1\end{array}$} & \multirow{3}{*}{$\begin{array}{c}\text { step } \\
2\end{array}$} & \multicolumn{6}{|c|}{ step 3} & \multirow{3}{*}{$\omega$} & \multirow{3}{*}{$\delta$} \\
\hline & & & \multicolumn{3}{|c|}{ process 1} & \multicolumn{3}{|c|}{ process 2} & & \\
\hline & & & state & UIO & check & state & 010 & check & & \\
\hline $1 \rightarrow 2$ & 0 & 0 & 2 & L1?nak & 0 & 1 & $L 2 ? m$ & 0 & 0 & 0 \\
\hline $2 \rightarrow 3$ & 0 & 0 & 2 & L1?nak & 0 & 2 & U2?nak & $\mathrm{O}$ & 0 & $\mathrm{O}$ \\
\hline $3 \rightarrow 4$ & 0 & 0 & 2 & L1?nak & 0 & 1 & $L 2 ? m$ & 0 & 0 & $\mathrm{O}$ \\
\hline $3 \rightarrow 5$ & 0 & 0 & 2 & L1?nak & 0 & 1 & $L 2 ? m$ & 0 & 0 & $\mathrm{O}$ \\
\hline $4 \rightarrow 1$ & 0 & 0 & 1 & U1?m & 0 & 1 & $L 2 ? m$ & 0 & 0 & $\mathrm{O}$ \\
\hline $5 \rightarrow 6$ & 0 & 0 & 3 & L1?nak & 0 & 1 & $L 2 ? m$ & 0 & 0 & 0 \\
\hline $6 \rightarrow 7$ & 0 & 0 & 3 & L1?nak & 0 & 2 & U2?nak & 0 & 0 & $\mathrm{O}$ \\
\hline $7 \rightarrow 8$ & 0 & 0 & 3 & L1?nak & 0 & 1 & $L 2 ? m$ & 0 & 0 & 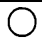 \\
\hline $8 \rightarrow 1$ & 0 & $\mathrm{O}$ & 1 & U1?m & 0 & 1 & $L 2 ? m$ & 0 & $\mathrm{O}$ & $\mathrm{O}$ \\
\hline $7 \rightarrow 9$ & 0 & 0 & 3 & L1?nak & 0 & 1 & $L 2 ? m$ & 0 & 0 & 0 \\
\hline $9 \rightarrow 1$ & 0 & 0 & 1 & U1?m & 0 & 1 & $L 2 ? m$ & 0 & $\mathrm{O}$ & 0 \\
\hline
\end{tabular}

Table 4 Error detection coverage of IP method.

\begin{tabular}{|c|c|c|c|c|c|c|}
\hline \multirow{2}{*}{ Method } & \multicolumn{2}{|c|}{ Trace method } & \multicolumn{2}{|c|}{ Simple method } & \multicolumn{2}{c|}{ IP method } \\
\cline { 2 - 7 } & $\omega$ & $\delta$ & $\omega$ & $\delta$ & $\omega$ & $\delta$ \\
\hline Completely detected errors & 8 & 5 & 8 & 5 & 11 & 11 \\
\hline Partial detected errors & - & 1 & - & 3 & - & 0 \\
\hline Not detected errors & 3 & 5 & 2 & 2 & 0 & 0 \\
\hline Transitions & 11 & 11 & 10 & 10 & 11 & 11 \\
\hline Error detection coverage (\%) & 73 & 50 & 80 & 65 & 100 & 100 \\
\hline
\end{tabular}

Table 5 Comparison of error detection coverages.

this system state graph. First, a method for generating test sequences effective for rough testing has been considered. Then a method for generating more strict and effective test sequences considering the independency of each process in interoperability testing by identifying the states of individual processes has been proposed.

An application for a simple protocol has shown that we can improve testing reliability in interoperability testing. In this example, it is concluded that all errors of the transition function and the output function of the system state graph can be detected. By using this method, reduction of costs of generating test sequences and improvement of reliability of testing can be expected for interoperability testing.

We are constructing a support system for test sequence generation based on the proposed method. As future works, a quantitative analysis of the amount of calculations and sequence lengths has to be done. Reduction of test sequences and SSG is also open problem.

\section{References}

[1] ISO : "OSI conformance testing methodology and framework", ISO 9646 (1989).

[2] S. Naito and M. Tsunoyama : Fault detection for sequential machines by transition tours", in Proc. IEEE Fault Tolerant Comput. Conf. (1981). 
[3] T. Chow : "Testing software design modeled by finite-state machines", IEEE Trans. Software Eng., SE-4, pp. 178-187 (1978).

[4] G. Gonenc: "A method for the design of fault detection experiment", IEEE Trans. Comput., C-19, pp. 551-558 (1970).

[5] K. Sabnami and A. Dahbura : A protocol test generation procedure", Computer Networks ISDN System, 15, pp. 285-297 (1988).

[6] D. Sidhu and T. Leung : "Formal method for protocol testing : a detailed study", IEEE Trans. Software Eng., SE-15, 4, pp. 413-426 (1989).

[7] H. Motteler, A. Chung and D. Sidhu : "Fault Coverage of UIO-based Method for Protocol Testing", Protocol Test Systems, VI, North-Holland, pp. 21-33 (1994)

[8] C. H. West: "General technique for communications protocol validation", IBM J.Res. \& Devel., 22, 4, pp. 394-404 (1978).

[9] D. Hogrefe : “Conformance testing based on formal methods", Proc. FORTE' '90, pp. 213-245 (1990).

[10] O. Rafiq and R. Castanet: "From conformance testing to interoperability testing", Proc. the 3rd International Workshop on Protocol Test Systems, pp. 371385 (1990).

[11] N. Arakawa and T. Soneoka : "A Test case generation method for concurrent programs", Proc. the 4th International Workshop on Protocol Test Systems, pp. 95-106 (1991).

[12] N. Okazaki, K. Takahashi, N. Shiratori and S. Noguchi : "Generation Method of Efficient Test Sequences from LOTOS Specifications”, Trans. IEICE Japan, J74B- I , 10, pp. 733-747 (1991).

[13] M. Park, N. Okazaki, M. Ohta, K. Takahashi, N. Shiratori and S. Noguchi : "Test Sequence Generation Method for Interoperability Testing Exploiting the Independency of Processes", Trans. IEICE Japan, J76-B- I , 3, pp. 264-274 (1993).

[14] N. Okazaki, M. Park, M. Ohta and K. Takahashi : "Test Sequence Generation for Multiprocess Communication Systems", Proc. the Asia-Pacific Conference on Communications, pp. 210-214 (1993). 